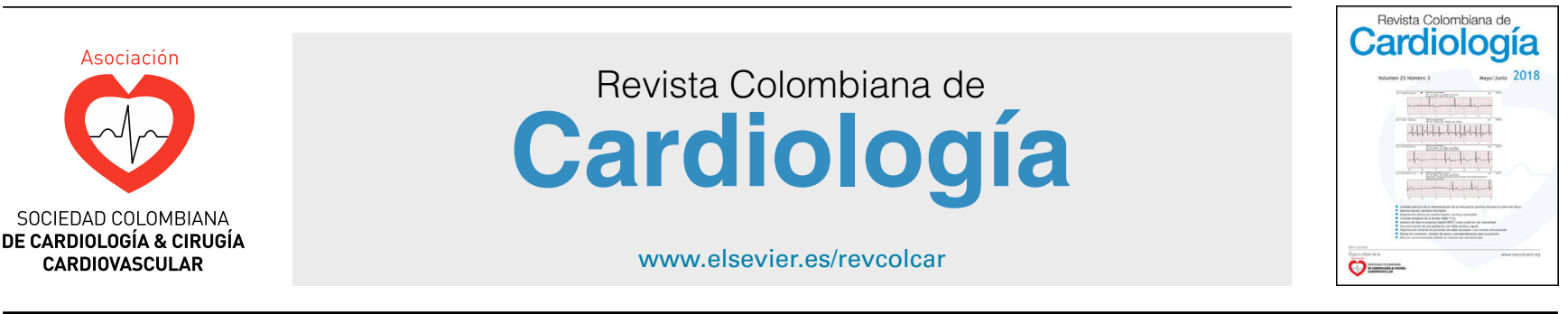

CARDIOLOGÍA DEL ADULTO - ARTÍCULO ORIGINAL

\title{
Concordancia entre ecoscopia realizada por médicos no cardiológos y ecocardiografía convencional
}

\author{
Carlos E. Guerrero ${ }^{\mathrm{a}, *, 1}$, Héctor M. Medina ${ }^{\mathrm{a}, 1}$, Óscar M. Pérez-Fernández ${ }^{\mathrm{b}, \mathrm{c}, 1}$, \\ Gonzalo Flórez ${ }^{\mathrm{a}}$, María Zorro ${ }^{\mathrm{a}, 1}$ y Gabriel Salazar ${ }^{\mathrm{d}, 1}$
}

\author{
a Departamento de Imágenes Cardiovasculares, Fundación Cardioinfantil - Instituto de Cardiología, Bogotá, Colombia \\ b Universidad del Rosario, Bogotá, Colombia \\ c Hospital Universitario Mayor Méderi, Bogotá, Colombia \\ ' Departamento de Cardiología y Laboratorio de Ecocardiografía, Fundación Cardioinfantil - Instituto de Cardiología, Bogotá, \\ Colombia
}

Recibido el 17 de febrero de 2018; aceptado el 15 de febrero de 2019

Disponible en Internet el 2 de julio de 2019

\section{PALABRAS CLAVE \\ Ecocardiografía; \\ Ultrasonografía; \\ Capacitación}

\begin{abstract}
Resumen
Introducción: el ultrasonido cardiaco enfocado ha sido propuesto como una aproximación útil para mejorar la toma de decisiones clínicas, permitiendo identificar rápidamente signos ultrasonográficos de una lista específica de diagnósticos potenciales.

Objetivo: evaluar un programa de entrenamiento para médicos sin experiencia en ecocardiografía a fin de realizar ultrasonido cardiaco enfocado por medio de un dispositivo portátil (ecoscopio).

Materiales y métodos: se compararon los resultados obtenidos mediante ecoscopia realizada por los médicos que recibieron el entrenamiento, con los obtenidos mediante ecocardiografía convencional realizada por cardiólogos expertos.

Métodos: un total de 5 médicos no cardiólogos incluyendo un estudiante de Medicina de último año, 2 residentes de Medicina Interna y 2 Intensivistas participaron en un curso de entrenamiento de cuatro semanas, dirigido por un Cardiólogo experto de nivel III así:

Primera semana: teoría y bases de ecocardiografía (3 horas diarias)

Segunda semana: teoría de la adquisición de imágenes. Hallazgos normales y anormales (50 estudios).

Tercera semana: manejo del ecoscopio (50 estudios).

Cuarta semana: recolección de datos.

Se incluyeron pacientes programados para ecocardiografía convencional en el Laboratorio de métodos no invasivos. A cada paciente se le realizaron dos exámenes. El primero consistió en una ecoscopia hecha por médico que recibió el entrenamiento y el segundo consistió en un ecocardiograma realizado por un cardiólogo experto.
\end{abstract}

\footnotetext{
* Autor para correspondencia.

Correo electrónico: carlosguerroroch@gmail.com (C.E. Guerrero).

1 Departamento de Imágenes Diagnósticas, Fundación Cardioinfantil - Instituto de Cardiología (FCI-IC), Bogotá-Colombia.
} 
Los parámetros ecocardiográficos evaluados fueron: fracción de eyección del ventrículo izquierdo, disfunción ventricular derecha, crecimiento auricular izquierdo, hipertensión pulmonar, enfermedad valvular cardiaca y derrame pericárdico. Por medio de análisis de concordancia (índice Kappa) se compararon los resultados encontrados en ecoscopia versus ecocardiografía. Resultados: de 221 estudios se obtuvo concordancia moderada en fracción de eyección del ventrículo izquierdo $(\kappa=0,541, p<0,000)$, función ventricular derecha $(\kappa=0,403, p<0,001)$, dilatación de la aurícula izquierda $(\kappa=0,413, p<0,001)$, valvulopatía mitral $(\kappa=0,466, p<0,001)$ y tricuspídea $(\kappa=0,437, p<0,001)$. La valvulopatía aórtica mostró un acuerdo débil. El derrame pericárdico y la hipertensión pulmonar tuvieron concordancia pobre y débil, respectivamente. Conclusiones: con un tiempo limitado de formación, los participantes sin experiencia previa en técnicas de ultrasonido y utilizando ecoscopia, alcanzaron un acuerdo moderado en la mayoría de las mediciones cuando se comparó con ecocardiografía convencional practicada por ecocardiografistas expertos. Es necesario un estudio con mayor número de participantes que determine el tiempo de formación ideal para obtener resultados comparables con ecocardiografía.

(c) 2019 Publicado por Elsevier España, S.L.U. en nombre de Sociedad Colombiana de Cardiología y Cirugía Cardiovascular. Este es un artículo Open Access bajo la licencia CC BY-NC-ND (http:// creativecommons.org/licenses/by-nc-nd/4.0/).

\section{KEYWORDS}

Cardiac Ultrasound (DeCS); Ultrasound (DeCS); Training (DeCS)

\section{Agreement between "echoscopy" performed by non-cardiologist physicians and conventional cardiac ultrasound}

\begin{abstract}
Introduction: Focused cardiac ultrasound has been proposed as a useful approach for improving clinical decision making, as well as to be able to rapidly identify the ultrasound signs of a specific list of potential diagnoses.

Objective: To evaluate a training program for physicians with no experience in cardiac ultrasound with the aim performing focused cardiac ultrasound using a portable device (echoscopy). Materials and methods: The results obtained from echoscopy performed by the physicians that received training were compared with those obtained with conventional cardiac ultrasound carried out by expert cardiologists.

A total of 5 non-cardiologist doctors, including 1 medical student, 2 Internal Medicine residents, and 2 from Intensive Medicine, took part in a four-week training course given by a Level III Cardiology specialist. The course included:

First week: Theory and basis of cardiac ultrasound ( 3 hours daily)

Second week: Theory of acquiring images. Normal and abnormal findings ( 50 studies).

Third week: handling of the echoscope (50 studies).

Fourth week: Data collection.
\end{abstract}

The study included patients scheduled for conventional cardiac ultrasound in the Non-Invasive Methods Laboratory. Two examinations were carried out on each patient. The first consisted of an echoscopy performed by a doctor that had received the training, and the second consisted of a cardiac ultrasound carried out by an expert cardiologist.

The ultrasound parameters evaluated were: left ventricular ejection fraction, right ventricular dysfunction, left atrial enlargement, pulmonary hypertension, cardiac valve disease, and pericardial effusion. The results found in echoscopy versus cardiac ultrasound were compared using concordance analysis (Kappa Index).

Results: The following results were obtained on the 221 studies performed: moderate agreement in left ventricular ejection fraction $(\kappa=0.541, p<.000)$, right ventricular function $(\kappa=0.403, p<.001)$, left atrial enlargement $(\kappa=0.413, p<.001)$, mitral valve and tricuspid valve disease $(\kappa=0.437, p<.001$ and $(\kappa=0.466, P<.001$, respectively). There was weak agreement with aortic valve disease. Pericardiac effusion and the presence of pulmonary hypertension had a poor and week agreement, respectively.

Conclusions: With a limited training period, the participants with no previous experience in ultrasound techniques and using echoscopy achieved a moderate agreement in the majority of measurements when compared with conventional cardiac ultrasound performed by experts in the technique. A study with a larger number of participants is required in order to determine the ideal training period to obtain results comparable with cardiac ultrasound.

(C) 2019 Published by Elsevier España, S.L.U. on behalf of Sociedad Colombiana de Cardiología y Cirugía Cardiovascular. This is an open access article under the CC BY-NC-ND license (http:// creativecommons.org/licenses/by-nc-nd/4.0/). 


\section{Introducción}

La evaluación morfológica y funcional cualitativa cardiaca a través de la ecocardiografía es una modalidad diagnóstica sin paralelo en la medicina, es menos costosa que otras técnicas en imágenes, y cuenta con la gran ventaja de poder ser aplicada en situaciones de emergencia por cardiólogos no ecocardiografistas, permitiendo así el diagnóstico y tratamiento oportunos de enfermedades que pueden requerir intervenciones inmediatas. La ecocardiografía convencional permite el análisis cualitativo y cuantitativo de la función sistólica biventricular y evalúa la competencia de todas las válvulas cardiacas, así como la severidad de un derrame pericárdico. Sin embargo, necesita de un dispositivo que no está disponible 24 horas en servicios de urgencias o unidades de cuidado intensivo y además requiere experticia en la adquisición y análisis de las imágenes ${ }^{1}$.

A diferencia de la ecocardiografía convencional, la ecoscopia cardiaca enfocada o FCU (sigla del inglés focused cardiac ultrasound), se hace con equipos portátiles (algunos de ellos de bolsillo), como complemento de la valoración física del paciente, permitiendo de este modo la visualización de estructuras y la cuantificación de parámetros que inciden de manera importante en el diagnóstico y tratamiento de cardiopatías en situaciones de emergencia ${ }^{2-4}$. Así, la ecoscopia cardiaca enfocada se define como una evaluación detallada del sistema cardiovascular, que es realizada por un médico que usa el ultrasonido junto con el examen físico, con el objetivo de reducir la lista de posibles diagnósticos diferenciales en un ámbito clínico determinado ${ }^{5}$.

El objetivo de este estudio fue evaluar un método de entrenamiento en ecoscopia cardiaca enfocada, de cuatro semanas de duración dictado a médicos no cardiólogos, a fin de determinar el grado de concordancia de parámetros ecocardiográficos básicos por medio de ecoscopia cardiaca enfocada (realizada a través de un dispositivo de bolsillo) versus ecocardiografía convencional hecha por ecocardiografistas expertos, a través de ecocardiógrafos de alta calidad.

\section{Materiales y métodos}

\section{Diseño del estudio}

Posterior a un periodo de capacitación de cuatro semanas a médicos no cardiólogos usando ecoscopia cardiaca enfocada, se realizó un estudio de concordancia diagnóstica entre ecoscopia cardiaca enfocada versus ecocardiografía convencional hecha por cardiólogos expertos de nivel III en el que se evaluaron cinco parámetros ecocardiográficos básicos. El estudio se llevó cabo en el laboratorio de métodos no invasivos de la Fundación Cardioinfantil - Instituto de Cardiología, durante los años 2013 a 2016.

\section{Capacitación de los participantes}

Previo a la toma de imágenes, todos los participantes recibieron capacitación dirigida por un ecocardiografista experto y por dos sonografistas, quienes prestaron asesoría y acompañamiento durante un entrenamiento de tres semanas, dividido en cuatro fases:

1. Primera semana: fundamentación teórica de los principios básicos ecocardiográficos, con una duración de tres horas diarias.

2. Segunda semana: observación de ecocardiogramas enseñando la técnica y el análisis de estudios normales y anormales alcanzando al menos 50 exámenes.

3. Tercera semana: entrenamiento sobre el manejo del ecoscopio, toma de imágenes, aproximación a la técnica de escaneo, ventanas ecocardiográficas e interpretación individual, siempre acompañado por el experto (50 exámenes).

4. Cuarta semana: toma de exámenes, ecoscopia cardiaca enfocada y ecocardiografía, recolección de la información.

\section{Participantes y variables ecocardiográficas en estudio}

Un estudiante de Medicina de último año (interno), dos residentes de Medicina Interna, dos Intensivistas (quienes utilizaron ecoscopia cardiaca enfocada) y los Cardiólogos ecocardiografistas del Laboratorio de Cardiología no invasiva de la institución (quienes usaron ecocardiógrafos convencionales de alta calidad).

Las imágenes y observaciones realizadas fueron ciegas a cada grupo, mientras que se evaluaron los parámetros de fracción de eyección del ventrículo izquierdo, función sistólica del ventrículo derecho, signos de hipertensión pulmonar, valvulopatías (tricuspídea, mitral o aórtica) y derrame pericárdico.

Las mediciones obtenidas por los participantes que realizaron ecoscopia cardiaca enfocada fueron completamente cualitativas. La fracción de eyección del ventrículo izquierdo se evaluó cualitativamente mediante la contractilidad del ventrículo izquierdo, en tanto que la función sistólica del ventrículo derecho se calculó comparando su contractilidad, así como el nivel de dilatación. El derrame pericárdico se definió como la presencia de una banda anecoica dentro de las hojas pericárdicas. La evaluación de las válvulas se hizo mediante flujo doppler color para determinación de regurgitación. La presencia de signos de hipertensión pulmonar se evaluó a través de medidas indirectas como la dilatación del ventrículo derecho, asociado con cambios morfológicos de sobrecarga de presión y volumen del ventrículo derecho en el septum interventricular.

Todas las medidas de ecoscopia cardiaca enfocada fueron hechas mediante un dispositivo de bolsillo V-scan ${ }^{\circledR}$ (GE Healthcare, Milwaukee, Wisconsin, EE.UU.), el cual está validado como herramienta diagnóstica para este $\operatorname{fin}^{7,8}$ y cuenta sólo con doppler color, por lo que la cuantificación de presiones sistólicas de ventrículo derecho u otras formas de cálculo de áreas y gradientes no se realizaron ${ }^{5}$, dada la ausencia de doppler espectral en este dispositivo.

Los ecocardiografistas expertos de nivel III pertenecientes al laboratorio de métodos no invasivos de la Fundación Cardioinfantil-Instituto de Cardiología utilizaron equipos de alta gama contemporáneos: VIVID 9 (General Electric 
System ${ }^{\circledR}$ ) y Philips le33 (Koninklijke Philips N.V., 2004 2017). La fase práctica de capacitación y el análisis de los datos se llevaron a cabo en la estación de trabajo ECHO PACK General Electric System ${ }^{\circledR}$.

Para lograr la adquisición de imágenes, tanto los participantes no cardiólogos, como los cardiólogos expertos utilizaron las siguientes cinco ventanas ecocardiográficas: i) eje largo paraesternal, ii) eje corto paraesternal, iii) apical 4 cámaras, iv), apical 5 cámaras, y v) subcostal.

\section{Población de estudio}

Pacientes consecutivos hospitalizados que ingresaban al laboratorio de métodos no invasivos de la Fundación Cardioinfantil-Instituto de Cardiología para la realización de un ecocardiograma (por cualquier razón). Todos los pacientes firmaron consentimiento informado para el estudio.

\section{Análisis estadístico}

Las variables categóricas se presentan como proporciones, en tanto que las variables continuas como medias y desviaciones estándar. Las variables ecocardiográficas analizadas fueron reportadas en categorías, de la siguiente manera:

- Fracción de eyección del ventrículo izquierdo: normal, levemente disminuida, moderadamente disminuida, severamente disminuida.

- Función sistólica del ventrículo derecho: normal, disfunción.

- Tamaño de la aurícula izquierda: normal o dilatada.

- Derrame pericárdico: leve, moderado, severo o ausencia.

- Hipertensión pulmonar: si o no.

- Valvulopatías: en esta variable, para cada una de las válvulas analizadas (mitral, tricúspide y aórtica) se reportaron las siguientes categorías: normal, insuficiencia, estenosis, doble lesión o prótesis.

Las siguientes variables fueron dicotomizadas para calcular los índices de concordancia, ya que no fue posible calcular la índice kappa para múltiples categorías en algunas variables policategóricas (las variables dicotómicas por naturaleza, no fueron sujeto de modificación):

Fracción de eyección del VI: normal/anormal

- Función ventricular derecha

Derrame pericárdico: si/no

- Hipertensión pulmonar: si/no

- Válvula mitral: normal/anormal

- Válvula tricúspide: normal/anormal

- Válvula aórtica: normal/anormal

Para cada una de las variables, se calculó en índice kappa (к). Los resultados con $\mathrm{p}<0,05$ fueron considerados significativos. La interpretación del índice kappa fue considerada de acuerdo con la clasificación internacionalmente aceptada así:

- Concordancia pobre: valores entre 0 y 0,2

- Concordancia débil: valores entre 0,21 y 0,40
Tabla 1 Características demográficas y clínicas de los pacientes

\begin{tabular}{ll}
\hline Característica & $\mathrm{n}=221$ \\
\hline Edad, media (DE) & $59,74(16,48)$ \\
Masculino, \% & 52,9 \\
IMC, media (DE) & $25,39(5,28)$ \\
HTA, \% & 43,9 \\
DM2, \% & 14,5 \\
EPOC, \% & 5 \\
Enfermedad coronaria, \% & 33 \\
Cardiopatía dilatada, \% & 9 \\
Enfermedad renal crónica, \% & 11,3 \\
Dislipidemia, \% & 15,8 \\
Cardiopatía valvular, \% & 8,6 \\
Ninguna comorbilidad, \% & 34,4 \\
\hline
\end{tabular}

IMC: índice de masa corporal, HTA: hipertensión arterial, DM2: diabetes mellitus tipo 2, EPOC: enfermedad pulmonar obstructiva crónica.

- Concordancia moderada: valores entre 0,41-0,60

- Concordancia buena: valores entre 0,61 y 0,80

- Concordancia excelente: valores mayores a 0,80

Los resultados de concordancia son presentados para la totalidad de pacientes evaluados y para cada uno de los 5 participantes que realizaron ecoscopia cardiaca enfocada por separado. Todos los análisis se hicieron en el programa SPSS v19.0.

\section{Resultados}

\section{Características poblacionales y de los participantes}

Los 5 participantes analizaron un total de 221 estudios (Interno: 27 estudios, residente de Medicina Interna 1: 47 estudios, residente de Medicina Interna 2: 58 estudios, Intensivista 1: 42 estudios, Intensivista 2: 47 estudios).

Las características clínicas de los pacientes se muestran en la tabla 1. La edad promedio de la población evaluada fue de 60 años, con una distribución homogénea por sexo y un índice de masa corporal (IMC) normal. Es de resaltar que hasta el $75 \%$ de los pacientes tenían al menos una comorbilidad, dentro de las cuales las más comunes fueron hipertensión arterial, diabetes mellitus tipo 2 y enfermedad coronaria.

\section{Análisis de concordancia}

Resultados globales $(n=221)$. En general, se encontró concordancia moderada entre la mayoría de los parámetros evaluados con ecoscopia cardiaca enfocada, en comparación con ecocardiografía convencional. Los resultados están incluidos en la tabla 2.

Función sistólica de los ventrículos izquierdo y derecho. Se encontró una concordancia moderada para la fracción de eyección del ventrículo izquierdo $(\kappa=0,474, p$ $<0,001$; dicotomizada $\kappa=0,541, p<0,001$ ) y para la función sistólica del ventrículo derecho $(\kappa=0,403, p<0,001)$. 
Tabla 2 Hallazgos ecocardiográficos generales encontrados por expertos y comparadores

\begin{tabular}{|c|c|c|c|c|c|}
\hline $\begin{array}{l}\text { Hallazgo ecocardiográfico } \\
(\mathrm{n}=221)\end{array}$ & & Experto & Comparador & Kappa & $\mathrm{p}$ \\
\hline \multirow{4}{*}{$\begin{array}{l}\text { Fracción de eyección del ventrículo } \\
\text { izquierdo }\end{array}$} & Levemente disminuida, $\%$ & 15,8 & 17,6 & 0,474 & $<0,001$ \\
\hline & Moderadamente disminuida, \% & 17,2 & 21,3 & & \\
\hline & Severamente disminuida, $\%$ & 11,3 & 13,1 & & \\
\hline & Normal, \% & 55,7 & 48,0 & & \\
\hline \multirow{4}{*}{$\begin{array}{l}\text { Fracción de eyección del ventrículo } \\
\text { izquierdo dicotomizada } \\
\text { Función ventrículo derecho }\end{array}$} & Normal, \% & 55,7 & 48,0 & 0,541 & $<0,001$ \\
\hline & Reducida, \% & 44,3 & 52,0 & & \\
\hline & Disfunción, \% & 15,8 & 16,7 & 0,403 & $<0,001$ \\
\hline & Normal, \% & 84,2 & 83,3 & & \\
\hline \multirow{6}{*}{$\begin{array}{l}\text { Aurícula } \\
\text { izquierda } \\
\text { Derrame } \\
\text { pericárdico }\end{array}$} & Dilatada, \% & 44,3 & 30,8 & 0,413 & $<0,001$ \\
\hline & Normal, \% & 55,7 & 69,2 & & \\
\hline & Leve, \% & 3,2 & 9,0 & 0,167 & 0,003 \\
\hline & Moderado, \% & 0 & 0 & & \\
\hline & Severo, \% & 0,5 & 0,5 & & \\
\hline & No, $\%$ & 96,4 & 90,5 & & \\
\hline \multirow{2}{*}{$\begin{array}{l}\text { Derrame pericárdico } \\
\text { dicotomizada }\end{array}$} & No, $\%$ & 96,4 & 90,5 & 0,163 & $<0,001$ \\
\hline & $\mathrm{Si}, \%$ & 3,6 & 9,5 & & \\
\hline \multirow[t]{2}{*}{ Hipertensión pulmonar } & No, $\%$ & 83,3 & 81,0 & 0,307 & $<0,001$ \\
\hline & $\mathrm{Si}, \%$ & 16,7 & 1,0 & & \\
\hline Válvula & Doble lesión, \% & 0,9 & 0,5 & 0,461 & $<0,001$ \\
\hline \multirow[t]{4}{*}{ mitral } & Prótesis, \% & 1,4 & 1,8 & & \\
\hline & Insuficiencia, \% & 30,3 & 25,3 & & \\
\hline & Estenosis, \% & 0,9 & 1,4 & & \\
\hline & Normal, \% & 66,5 & 71,1 & & \\
\hline \multirow{5}{*}{$\begin{array}{l}\text { Válvula mitral } \\
\text { dicotomizada } \\
\text { Válvula tricúspide }\end{array}$} & Anormal, \% & 33,5 & 28,9 & 0,466 & $<0,001$ \\
\hline & Normal \% & 66,5 & 71,1 & & \\
\hline & Insuficiencia, \% & 24 & 17,2 & 0,437 & $<0,001$ \\
\hline & Estenosis, \% & 0 & 0 & & \\
\hline & Normal, \% & 76 & 82,8 & & \\
\hline \multirow[t]{2}{*}{ Válvula tricúspide dicotomizada } & Anormal, \% & 24,0 & 17,2 & 0,437 & $<0,001$ \\
\hline & Normal, \% & 76,0 & 82,8 & & \\
\hline \multirow[t]{5}{*}{ Válvula aórtica } & Doble lesión, \% & 0 & 0 & NA & NA \\
\hline & Presencia de prótesis, \% & 1,4 & 1,4 & & \\
\hline & Insuficiencia, \% & 17,6 & 10,4 & & \\
\hline & Estenosis, \% & 0,5 & 0,9 & & \\
\hline & Normal, \% & 80,5 & 87,3 & & \\
\hline \multirow[t]{2}{*}{ Válvula aórtica dicotomizada } & Anormal, \% & 19,5 & 12,7 & 0,218 & 0,001 \\
\hline & Normal, \% & 80,5 & 87,3 & & \\
\hline
\end{tabular}

Evaluación de la aurícula izquierda. El volumen de la aurícula izquierda tuvo una concordancia moderada $(\kappa=0,413, \mathrm{p}<0,000)$.

Valvulopatías. En su mayoría presentaron concordancia moderada (mitral: $\kappa=0,461, \mathrm{p}<0,001$; dicotomizada $\kappa=0,466, p<0,001$ ), (tricúspide: $\kappa=0,437, p<0,001$; dicotomizada $\kappa=0,437, p<0,001$ ); sin embargo, la valvulopatía aórtica mostró concordancia débil (dicotomizada $\kappa=0,218$, $p=0,001)$.

Derrame pericárdico e hipertensión pulmonar. Para estos parámetros se encontró una concordancia pobre y débil, respectivamente (derrame pericárdico: $\kappa=0,167, p$ 0,003; dicotomizada $\kappa=0,163$, p 0,006), (hipertensión pulmonar: $\kappa=0,307, p<0,001$.
Evaluación por participante. Las tablas para cada uno de los participantes se presentan en material suplementario (tablas 3 a 5, material suplementario).

Interno (tabla 3 material suplementario). Este participante tuvo concordancia con valores de índice Kappa entre buena a excelente en los parámetros de funcionalidad del ventrículo izquierdo y derecho (fracción de eyección del ventrículo izquierdo $\kappa=0,788$, $p<0,001$; dicotomizada $\kappa=0,886, p<0,001$ y ventrículo derecho $\kappa=0,649$, $\mathrm{p}<0,001)$. Los valores de dilatación de la aurícula izquierda tuvieron una concordancia igualmente buena. Para las valvulopatías, la concordancia en la mayoría fue moderada a excepción de la válvula tricúspide, donde fue débil $(\kappa=0,198, p 0,085)$. 
Residentes de Medicina Interna (tabla 4, material suplementario). Para los parámetros de fracción de eyección del ventrículo izquierdo, función sistólica del ventrículo derecho y evaluación de la aurícula izquierda se encontró un grado de acuerdo moderado (fracción de eyección del ventrículo izquierdo $\kappa=0,502, p<0,001$, dicotomizada $\kappa=0,568, p<0,001$; función sistólica del ventrículo derecho $\kappa=0,446, p<0,001)$.

Las valvulopatías aórtica y tricúspide en este grupo de examinadores tuvieron concordancia pobre sin alcanzar significancia estadística. La excepción en este grupo fue la insuficiencia mitral, que alcanzó una concordancia débil a moderada $(\kappa=0,407, p<0,001$, dicotomizada $\kappa=0,386, p$ $<0,001)$.

La concordancia fue pobre para evaluación del derrame pericárdico sin alcanzar significancia estadística $(\kappa=0,063)$.

Intensivistas (tabla 5, material suplementario). En este grupo, el mayor grado de acuerdo estuvo en la detección de valvulopatías donde se encontró buena concordancia para la valvulopatía tricuspídea $(\kappa=0,71, p<0,001$, dicotomizada $\kappa=0,711, p<0,001)$ y concordancia moderada para la valvulopatía mitral $(\kappa=0,519, p<0,001)$. La excepción nuevamente fue la valvulopatía aórtica, que demostró una concordancia débil.

Para los valores de fracción de eyección del ventrículo izquierdo y función sistólica del ventrículo derecho los resultados de concordancia fueron débiles. Lo mismo ocurrió para los parámetros de derrame pericárdico, aurícula izquierda e hipertensión pulmonar.

\section{Discusión}

Este estudio demostró que médicos no cardiólogos (un interno, dos residentes de Medicina interna y dos intensivistas) con un entrenamiento de cuatro semanas en ecoscopia cardiaca enfocada, lograron una concordancia moderada para los parámetros fracción de eyección del ventrículo izquierdo, función sistólica del ventrículo derecho y evaluación volumétrica cualitativa de la aurícula izquierda, al comparar con los resultados de ecocardiografía convencional realizada por un cardiólogo experto.

En la evaluación de las válvulas cardiacas se observó que para las válvulas mitral y tricúspide, el grado de acuerdo llegó a ser moderado, sin embargo, la válvula aórtica no tuvo estos resultados. La pobre concordancia observada en la válvula aórtica, se debe a que su aproximación ecocardiográfíca requiere mayor pericia y el uso de diferentes proyecciones y planos ultrasonográficos para lograr una evaluación óptima. Así mismo, los métodos de evaluación para determinar su severidad (sean directos o indirectos), generalmente requieren del uso de doppler espectral, evaluación del color en eje largo paraesternal y mediciones volumétricas específicas sobre el ventrículo izquierdo ${ }^{9}$, que se salen del alcance de la ecoscopia cardiaca enfocada. En este estudio se omitió la evaluación de la válvula pulmonar, en consideración a que la aproximación a esta válvula por ecocardiografía requiere mayor experiencia, así como el uso de doppler espectral, que no estaba disponible en el dispositivo utilizado en ecoscopia cardiaca enfocada.

La baja concordancia observada en las valvulopatías concuerda con las guías actuales, donde reiteran que añadirle doppler color a la ecoscopia cardiaca enfocada requiere mayor tiempo de entrenamiento enfocado en esta técnica, para lograr una adquisición e interpretación adecuada de estas funciones. Además, estas capacidades normalmente no son necesarias para el alcance práctico de la ecoscopia cardiaca enfocada ${ }^{5}$.

Se halló concordancia débil o pobre en parámetros que requieren más experticia, mediciones directas y diferentes proyecciones para lograr una evaluación completa, como es el caso de la hipertensión pulmonar. Para su detección se precisan signos ecocardiográficos cualitativos, como por ejemplo el aplanamiento del septum interventricular, los cuales son subjetivos y tienen baja sensibilidad. Adicionalmente, para una aproximación adecuada a la cuantificación de la hipertensión pulmonar, se requieren mediciones directas de la velocidad de la insuficiencia tricuspídea y el cálculo de la presión de la aurícula derecha con el índice de vena cava, que están por fuera del alcance de la ecoscopia cardiaca enfocada.

Otro es el caso del derrame pericárdico, en donde la baja prevalencia de pacientes con esta alteración, impidió el cálculo de los índices de concordancia. Sólo se encontró un caso de derrame pericárdico severo en toda la población evaluada, el cual fue detectado tanto por ecoscopia cardiaca enfocada, como por ecocardiografía convencional. Es probable que con una mayor prevalencia de derrame pericárdico, la concordancia sea alta debido a que es un parámetro fácilmente evaluable por cualquiera de los dos métodos en estudio (ecoscopia cardiaca enfocada y ecocardiografía convencional) y no se requiere mayor entrenamiento para la identificación de esta alteración.

Este estudio se basó en un programa de entrenamiento corto, en el cual durante la última semana se realizó la adquisición e interpretación de 50 registros a través de ecoscopia cardiaca enfocada, sobre bases teóricas y observación de ecocardiogramas convencionales. Con esto se pretende demostrar que, con un entrenamiento básico, los médicos sin ninguna experiencia previa con el ultrasonido, pueden utilizar la ecoscopia cardiaca enfocada como extensión del examen físico con el fin de identificar alteraciones básicas cardiovasculares, en un centro cardiovascular de alto volumen. Es de resaltar que en el estudio se utilizó la ecoscopia cardiaca enfocada y no la ecocardiografía limitada. La diferencia entre estas dos modalidades radica en que el equipo, para la realización de ecografía limitada, cuenta con todos los parámetros funcionales y de medición (doppler tisular, espectral, modo $M$, doppler color, contraste) que se incluyen en un estudio ecocardiográfico convencional y permite cuantificaciones avanzadas, con un protocolo predeterminado y realizado por un ecocardiografista nivel II o III. La ecoscopia cardiaca enfocada, por el contrario, sólo permite la adquisición de imágenes con interpretación cualitativa sin medición alguna de parámetros ecocardiográficos. El término "limitada"' se refiere al análisis de un número específico de imágenes en un protocolo establecido, mientras que "enfocado" alude a que intenta responder una pregunta específica identificando la presencia o ausencia de uno o varios hallazgos específicos mediante un protocolo definido y establecido de adquisición de imágenes basado en el alcance y la experticia del operador ${ }^{5}$.

Un estudio previo, en el que se evaluó un programa de entrenamiento mínimo realizado por Bustaman, et $a l^{10}$. 
analizó parámetros similares e incluyó residentes de Medicina de Emergencias. Los resultados obtenidos mostraron buena concordancia en la evaluación de la función sistólica ventricular izquierda ( $\kappa=0,79$ IC95\% 0,773-0,842), con resultados más débiles en cuanto a derrame pericárdico e índice de vena cava inferior. Es de resaltar que los residentes de Emergencias tienen entrenamiento previo en ecocardiografía durante su curriculum académico ${ }^{10,11}$. Esta es una diferencia importante con el presente estudio, pues ninguno de los participantes tenía conocimiento previo de ultrasonido o ecocardiografía. Por otro lado, el equipo utilizado en el estudio de Bustaman (Logiq-e General Electronics con un transductor cardiaco de $2-3.6 \mathrm{MHz}$ phased array) es de una gama más alta y posee características que incluyen doppler espectral y continuo, modo $M$, imágenes con armónicos, así como todos los paquetes de mediciones; además tiene mejor resolución de pantalla, lo cual confiere la posibilidad de hacer ecocardiografía limitada. En el presente trabajo, la portabilidad del equipo, las observaciones realizadas sin mediciones lineares y sin ningún tipo de cuantificación están más acordes con las recomendaciones actuales sobre el alcance que se quiere lograr con la ecoscopia cardiaca enfocada, hecho que podría explicar la disparidad de los resultados, como es el caso de las comparaciones que requirieron el uso de doppler color y espectral.

Otros estudios como el de Mjølstad, et $a l^{12}$., han intentado realizar comparaciones en residentes médicos (residentes de clínicas médicas) con mejores resultados en la evaluación de fracción de eyección del ventrículo izquierdo, función sistólica del ventrículo derecho volumen de aurícula derecha y derrame pericárdico. Sin embargo, los participantes recibieron un entrenamiento formal en ecocardiografía con una duración de tres meses, mucho más prolongado, que a la luz de las guías actuales de formación ecocardiográfica, equivale a adquirir un nivel I de entrenamiento ${ }^{5,6,13,14}$.

Teniendo en cuenta la diversidad entre los tipos de entrenamiento, vale la pena recordar que la función para la cual se utiliza la ecoscopia cardiaca enfocada no es más que ser una herramienta dirigida a mejorar la exploración física, donde el especialista con conocimiento básico sobre la técnica ecocardiográfica, realiza el estudio del paciente para obtener orientación acerca del diagnóstico y la necesidad de tratamiento inmediato, particularmente en los servicios de emergencia y cuidado intensivo. Entre los diagnósticos diferenciales más importantes que permite detectar la ecoscopia cardiaca enfocada se incluyen la disfunción ventricular derecha o izquierda y la presencia de valvulopatía severa o derrame pericárdico ${ }^{11}$.

Hasta el momento no hay un consenso o recomendación oficial en cuanto a las competencias o el tiempo de entrenamiento que se deben tener en cuenta para la interpretación adecuada de la ecoscopia cardiaca enfocada. Las opiniones actuales indican que dichas competencias deben ser similares a las estipuladas en la guía de entrenamiento en competencias en ecocardiografía ${ }^{6,13,14}$. El presente estudio apoya estas consideraciones ya que un entrenamiento acortado, como el que se llevó a cabo, logró niveles de acuerdo sólo hasta moderados en algunos de los parámetros y débiles en otros, sin obtener una buena o excelente concordancia en ningún caso.

En estudios similares por Mjølstad et al ${ }^{12}$, Lucas et al. ${ }^{15}$ y Leta et al. ${ }^{16}$, que incluyeron tiempos de entrenamiento más prolongando y personal en entrenamiento cardiológico, se obtuvieron mejores resultados en parámetros de función ventricular izquierda; sin embargo, los resultados para valvulopatías y función ventricular derecha fueron similares a los que se plantean aquí.

Un ejemplo particular del tiempo de entrenamiento como factor determinante en los resultados es el caso del estudio de Mjølstad et al. ${ }^{12}$, donde los residentes fueron contratados por el laboratorio de ecocardiografía durante el programa de entrenamiento, lo que permitió completar los tres meses de entrenamiento.

El tiempo disponible para el entrenamiento de los participantes fue unas de las limitantes, ya que tanto los residentes como el interno, tenían una carga asistencial que les impedía disponer de tiempo libre para la investigación. Fue necesario que realizaran una rotación electiva en el laboratorio de ecocardiografía para poder participar. Esto explica el tiempo corto en su entrenamiento y la dificultad en la consecución de los participantes.

Para evitar la posibilidad de sesgos, la evaluación de ecoscopia cardiaca enfocada siempre se realizó previo al estudio ecocardiográfico y ambos, tanto participantes como ecocardiografistas, fueron ciegos a los resultados.

La propuesta de un estudio que determine el tiempo adecuado de entrenamiento requerido para la interpretación correcta de ecoscopia cardiaca enfocada es imperativa, considerando que los lineamientos actuales apoyan que reciban un entrenamiento similar al recibido por ecocardiografistas de nivel I. Esto resulta problemático dado que el alcance de la ecoscopia cardiaca enfocada, como se ha mencionado previamente, no es entrenar a un experto en ecocardiografía, sino prestar una herramienta que sirva de extensión al examen físico en situaciones donde tal información tiene un gran impacto, como lo pueden ser el servicio de urgencias o las unidades de cuidado intensivo.

\section{Conclusiones}

Con un tiempo limitado de entrenamiento, mediante el uso de ecoscopia cardiaca enfocada, los participantes lograron una concordancia moderada para los parámetros de función ventricular izquierda, función ventricular derecha, valvulopatía mitral y tricúspide, concordancia pobre y débil para derrame pericárdico y valvulopatía aórtica. Este estudio también sugiere que para mejorar el grado de acuerdo en otras mediciones o para una aproximación completa, es factible que se necesite un tiempo de entrenamiento idealmente más prolongado. Es necesario un estudio que pruebe con validez adecuada el tiempo de entrenamiento requerido para realizar correctamente ecoscopia cardiaca enfocada.

\section{Reconocimientos}

A todos los pacientes y a cada uno de los participantes (interno, residentes e intensivistas) quienes voluntariamente aceptaron hacer parte de este estudio, sin los cuales no hubiera sido posible llevarlo a cabo. 


\section{Financiación}

Recursos propios de los investigadores y del Departamento de Cardiología de la Fundación Cardioinfantil - Instituto de Cardiología.

\section{Conflicto de intereses}

Ninguno.

\section{Anexo. Material adicional}

Se puede consultar material adicional a este artículo en su versión electrónica disponible en doi:10.1016/j.rccar.2019.02.003.

\section{Bibliografía}

1. García-Vicente E, Campos-Nogué A, Gobernado MM. Ecocardiografía en la Unidad de Cuidados Intensivos. Med Intensiva. 2008;32:236-47.

2. Seward JB, Douglas PS, Erbel R, Kerber Rem Kronzon I, Rakowski $\mathrm{H}$, et al. Hand-carried cardiac ultrasound ( $\mathrm{HCO}$ ) device: recommendations regarding new technology. A report from the Echocardiography Task Force on New Technology of Nomenclature and Standars Committee of the American Society of Echocardiography. J Am Soc Echocardiogr. 2002;15:369-73.

3. Senior R, Chambers J. Portable echocardiography: a review. $\mathrm{Br}$ J Cardiol. 2006;13:185-90.

4. Labovitz AJ, Noble VE, Biering M, Goldstein SA, Jones R, Kort $S$, et al. Focused cardiac ultrasound in the emergent setting: a consensus statement of the American Society of Echocardiography and American College of Emergency Physicians. J Am Soc Echocardiogr. 201;23(12):1225-30.

5. Spencer KT, Kimura BJ, Korcarz CE, Pellikka PA, Rahko PS, Siegel RJ. Focused cardiac ultrasound: recommendations from the American Society of Echocardiography. J Am Soc Ecochardior. 2013;26:567-81.

6. Ryan T, Armstrong WF, Khandheria BK. Task Force 4: training in echocardiography endorsed by the American Society of Echocardiography. J Am Coll Cardiol. 2008;51:361-7.
7. Cardim N, Fernandez Golfin C, Ferreira D, Aubele A, Toste JCobos MA, et al. Usefulness of a new miniaturized echocardiographic system in outpatient cardiology consultations as an extention of physical examination. J Am Soc Echocardiogr. 2011;24:117-24.

8. Liebo MJ, Israel RL, Lillie EO, Smith MR, Rubenson DS, Topol EJ. Is pocket mobile echocardiography the next-generation stethoscope? A cross-sectional comparison of rapidly acquired images with standard transthoracic echocardiography. Ann Intern Med. 2011;155:33-8.

9. Zoghbi W, Adams D, Bonow RO, Enriquez-Sarano M, Foster E, Grayburn PA, et al. Recommendations for Noninvasive Evaluation of Native Valvular Regurgitation: A Report from the American Society of Echocardiography Developed in Collaboration with the Society for Cardiovascular Magnetic Resonance. J Am Soc Echocardiogr. 2017;30:303-71.

10. Bustaman A, Noor Ashar M, Singh Veriah R, Arumugam K, Loch A. Performance of emergency physicians in point-of-care echocardiography following limited training. Emerg Med J. 2014;31:369-73.

11. Ultrasound Guidelines: Emergency, Point-of-Care and Clinical Ultrasound Guidelines in Medicine. Ann Emerg Med. 2017;69(5):e27-e54.

12. Mjølstad OC, Andersen GN, Dalen H, Graven T, Skjetne, Kleinau JO, et al. Feasibility and reliability of point-of-care pocket-size echocardiuography performed by medical residents. Eur Heart J Cardiovasc Imaging. 2013;14:1195-202.

13. Barba Cosials J, Pérez de Isla L. Ecocardiografia fuera del ámbito de la Cardiología Posición y recomendaciones de la Sociedad Española de Cardiología. Rev Esp Cardiol. 2016;69: 644-6.

14. La ecocardiografía fuera del ámbito de la Cardiología: recomendaciones de la Sociedad Española de Cardiología para un uso adecuada de los equipos de ecocardiografía de bolsillo. Rev Esp Cardiol. 2014:1-13.

15. Lucas BP, Candotti C, Margeta B, Evans AT, Mba B, Baru J, et al. Diagnostic accuracy of hospitalist-performed hand-carried ultrasound echocardiography after a brief training program. J Hosp Med. 2009;4:340-9.

16. Leta R, Carreras F, Borrás X, Planas F, Pujadas S, Pons-Lladó G. Utilidad de un equipo de ecocardiografía portátil en un servicio de urgencias generales. Med Clin (Barc). 2003;121: $178-80$. 